\title{
A DICHOTOMY FOR FATOU COMPONENTS OF POLYNOMIAL SKEW PRODUCTS
}

\author{
ROLAND K. W. ROEDER
}

\begin{abstract}
We consider polynomial maps of the form $f(z, w)=(p(z), q(z, w))$ that extend as holomorphic maps of $\mathbb{C P}^{2}$. Mattias Jonsson introduces in "Dynamics of polynomial skew products on C $\mathbf{C}^{2}$ " [Math. Ann., 314(3): 403$447,1999]$ a notion of connectedness for such polynomial skew products that is analogous to connectivity for the Julia set of a polynomial map in one-variable. We prove the following dichotomy: if $f$ is an Axiom-A polynomial skew product, and $f$ is connected, then every Fatou component of $f$ is homeomorphic to an open ball; otherwise, some Fatou component of $F$ has infinitely generated first homology.
\end{abstract}

\section{INTRODUCTION}

We consider the dynamics of mappings $f: \mathbb{C}^{2} \rightarrow \mathbb{C}^{2}$ of the form

$$
f(z, w)=(p(z), q(z, w))
$$

where $p$ and $q$ are polynomials. It will be convenient to assume that $\operatorname{deg}(p)=$ $\operatorname{deg}(q)=d$ and $p(z)=z^{d}+O\left(z^{d-1}\right)$ and $q(z, w)=w^{d}+O_{z}\left(w^{d-1}\right)$ so that $f$ extends as a holomorphic mapping $f: \mathbb{C P}^{2} \rightarrow \mathbb{C P}^{2}$. (Throughout this paper we will assume that $d \geq 2$.) Since $f$ preserves the family of vertical lines $\{z\} \times \mathbb{C}$, one can analyze $f$ via the collection of one variable fiber maps $q_{z}(w)=q(z, w)$, for each $z \in \mathbb{C}$. In particular, one can define fiber-wise filled Julia sets $K_{z}$ and Julia sets $J_{z}$ that are analogous to their one-dimensional counterparts. For this reason, polynomial skew products provide an accessible generalization of one variable dynamics to two variables. They have been previously studied by many authors, including Heinemann in [6] and [7], Jonsson in [12, DeMarco-Hruska in [2], and Hruska together with the author of this note in [8].

A more general situation in which the base is allowed to be an arbitrary compact topological space, while the vertical fibers are copies of $\mathbb{C}$, has been considered by Sester in [17] and [18. Meanwhile, generalization to semigroups of polynomial (and rational) mappings of the Riemann sphere has been studied extensively by Hinkkanen, Martin, Ren, Stankewitz, Sumi, Urbański, and many others - we refer the reader to the excellent bibliography from 21 for further references.

Received by the editors May 12, 2010 and, in revised form, January 1, 2011, and January 2, 2011.

2010 Mathematics Subject Classification. Primary 32H50; Secondary 37F20, 57R19.

Key words and phrases. Fatou components, linking numbers, closed currents, holomorphic motions.

Research was supported in part by startup funds from the Department of Mathematics at IUPUI. 
The following notion is introduced in [12] as an analogy with polynomial maps of one variable.

Definition 1.1 (Jonsson). A polynomial skew product $f$ is connected if $J_{p}$ is connected and $J_{z}$ is connected for all $z \in J_{p}$.

Here, $J_{p}$ is the Julia set for the "base map" $z \mapsto p(z)$. Jonsson proves in [12, Sec. $6]$ that if $f$ is a connected polynomial skew product, then the support $J_{2}:=\operatorname{supp} \mu$ of its measure of maximal entropy $\mu$ (see $\$ 2$ below) is connected, the Hausdorff dimension of $\mu$ is 2 , and the associated Lyapunov exponents are precisely $\log d$. (Note that $J_{2}$ can be connected even if $f$ is a disconnected polynomial skew product; see [2, Lemma 5.5].)

The Fatou set $U(f)$ of a holomorphic map $f: \mathbb{C P}^{2} \rightarrow \mathbb{C P}^{2}$ is the maximal open set in $\mathbb{C P}^{2}$ on which the sequence of iterates is normal and the Julia set is its complement: $J(f):=\mathbb{C P}^{2} \backslash U(f)$. In this note, we prove the following.

Theorem 1.2. Suppose that $f$ is an Axiom-A polynomial skew product. If $f$ is connected, then every Fatou component of $f$ is homeomorphic to an open ball. Otherwise, some Fatou component of $f$ has infinitely generated first homology.

The Axiom-A assumption ensures hyperbolicity of $f$ on the non-wandering set; see, for example, [12, Sec. 8] and [2]. Using the equivalence between (1) and (2) from Theorem 2.1 (below), connectivity of $f$ can be expressed in terms of "escape of the critical locus", so Theorem 1.2 is an analog of the fundamental dichotomy from polynomial dynamics in one variable (see, for example [15, Theorem 9.5]).

Corollary 1.3. If $f$ is an Axiom-A polynomial skew product for which $J_{2}=\operatorname{supp} \mu$ is disconnected, then some Fatou component of $f$ has infinitely generated first homology.

We will discuss in Remark 4.4 the natural question of whether Theorem 1.2 holds if $f$ is not Axiom-A. We first show that some hypothesis of hyperbolicity is needed, by providing an example of a disconnected product mapping that is not Axiom-A, whose Fatou set has a single component that is homeomorphic to an open ball. We will then show that the proof of Theorem 1.2 that is presented in Section 4 continues to hold if we replace Axiom-A with slightly weaker conditions.

\section{BACKGROUND ON POLYNOMIAL SKEW PRODUCTS}

We present some background on polynomial endomorphisms of $\mathbb{C P}^{2}$ from [3], 9], 22, [19, and 1] and on polynomial skew products from [12.

Let $f: \mathbb{C}^{2} \rightarrow \mathbb{C}^{2}$ be given by

$$
f(z, w)=(p(z, w), q(z, w)),
$$

with $p$ and $q$ polynomials of degree $d \geq 2$, and let $p_{d}$ and $q_{d}$ be the homogeneous parts of $p$ and $q$, respectively, of the maximal degree $d$. Such a mapping extends as a holomorphic mapping $f: \mathbb{C P}^{2} \rightarrow \mathbb{C P}^{2}$ if and only if $p_{d}(z, w)=0=q_{d}(z, w)$ implies that $(z, w)=(0,0)$. Any holomorphic mapping $f: \mathbb{C P}^{2} \rightarrow \mathbb{C P}^{2}$ that is obtained in this way is called a polynomial endomorphism of $\mathbb{C P}^{2}$.

Suppose that $f: \mathbb{C}^{2} \rightarrow \mathbb{C}^{2}$ extends as a polynomial endomorphism of $\mathbb{C P}^{2}$. The (affine) Green's function

$$
G(z, w)=\lim \frac{1}{d^{n}} \log _{+}\left\|f^{n}(z, w)\right\|, \text { where } \log _{+}:=\max \{\log , 0\},
$$


is a plurisubharmonic (PSH) function having the property that $(z, w) \in \mathbb{C}^{2}$ is in $U(f)$ if and only if $G(u, v)$ is pluriharmonic $(\mathrm{PH})$ in a neighborhood of $(z, w)$. The Green's current $T:=\frac{1}{2 \pi} d d^{c} G$ is a closed positive $(1,1)$ current on $\mathbb{C}^{2}$ with $J(f):=\operatorname{supp} T$ and $U(f):=\mathbb{C}^{2} \backslash \operatorname{supp} T$.

If $f$ is a polynomial skew product, i.e. $p(z, w) \equiv p(z)$, there is a refinement of this description. The base map $p(z)$ has a Julia set $J_{p} \subset \mathbb{C}$ and, similarly, a Green's function $G_{p}(z):=\lim _{n \rightarrow \infty} \frac{1}{d^{n}} \log _{+}\left\|p^{n}(z)\right\|$. Furthermore, one can define a fiber-wise Green's function by:

$$
G_{z}(w):=G(z, w)-G_{p}(z) .
$$

For each fixed $z, G_{z}(w)$ is a subharmonic function of $w$ and one defines the fiber-wise Julia sets by $K_{z}:=\left\{G_{z}(w)=0\right\}$ and $J_{z}:=\partial K_{z}$.

The extension of $f$ to $\mathbb{C P}^{2}$ is given by

$$
f([Z: W: T])=\left[P(Z, T): Q(Z, W, T): T^{d}\right],
$$

where $P(Z, T)$ and $Q(Z, W, T)$ are the homogeneous versions of $p$ and $q$. The point $[0: 1: 0]$ that is "vertically at infinity" with respect to the affine coordinates $(z, w)$ is a totally invariant super-attracting fixed point and $(z, w) \in W^{s}([0: 1: 0])$ if and only if $w \in \mathbb{C} \backslash K_{z}$.

The action of $f$ on the line at infinity $\Pi:=\{T=0\}$ is given by the polynomial map $f_{\Pi}(u)=q_{d}(1, u)$, where $u=w / z$ and $q_{d}(z, w)$ is the homogeneous part of $q$ of maximal degree $d$. As usual, one can consider the associated Julia sets $K_{\Pi}$ and $J_{\Pi}$.

One can extend the Green's current $T$ as a closed positive $(1,1)$ current on all of $\mathbb{C P}^{2}$ satisfying $f^{*} T=d \cdot T$ and having the property that $J(f)=\operatorname{supp} T$ and $U(f)=\mathbb{C P}^{2} \backslash \operatorname{supp} T$. Moreover, the wedge product $\mu:=T \wedge T$ is a measure satisfying $f^{*} \mu=d^{2} \cdot \mu$, which happens to be the measure of maximal entropy for $f$. It is customary to define a second Julia set $J_{2} \equiv J_{2}(f):=\operatorname{supp} \mu \subset J(f)$.

If $f=(p, q)$ is a polynomial skew product, then [12, Cor. 4.4] gives

$$
J_{2}=\overline{\bigcup_{z \in J_{p}}\{z\} \times J_{z}} .
$$

The following is (an excerpt from) Theorem 6.5 from [12]:

Theorem 2.1 (Jonsson). The following are equivalent:

(1) $f$ is connected.

(2) $C_{p} \subset K_{p}$ and $C_{z} \subset K_{z}$, for all $z \in J_{p}$.

(3) $J_{p}$ is connected, $J_{\Pi}$ is connected, and $J_{z}$ is connected, for all $z \in \mathbb{C}$.

(4) $C_{p} \subset K_{p}, C_{\Pi} \subset K_{\Pi}$, and $C_{z} \subset K_{z}$, for all $z \in \mathbb{C}$.

Here $C_{p}$ and $C_{\Pi}$ are the critical points of $p$ and $f_{\Pi}$, while $C_{z}:=\{(z, w): \partial q / \partial w$ $=0\}$.

The following appears in [8]:

Theorem 2.2 (Hruska-R). If $J_{z}$ is connected for every $z \in J_{p}$, then $W^{s}([0: 1: 0])$ is homeomorphic to an open ball. Otherwise, the first homology $H_{1}\left(W^{s}([0: 1: 0])\right)$ is infinitely generated.

The proof that $W^{s}([0: 1: 0])$ is homeomorphic to a ball is an application of $G_{z}(w)$ as a type of Morse function, while the latter uses non-trivial linking numbers between closed loops in $W^{s}([0: 1: 0])$ and the Green's current $T$. 


\section{BACKGROUND ON LINKING \\ With a Closed positive $(1,1)$ CURREnt IN $\mathbb{C P}^{2}$}

We present a brief summary (without proofs) of material from [8, §3]; see also [16].

Any closed positive $(1,1)$ current $S$ on a complex manifold $N$ can be described using an open cover $\left\{U_{i}\right\}$ of $N$ together with PSH functions $v_{i}: U_{i} \rightarrow[-\infty, \infty)$ that are chosen so that $S=d d^{c} v_{i}$ in each $U_{i}$. The functions $v_{i}$ are called local potentials for $S$ and they are required to satisfy the compatibility condition that $v_{i}-v_{j}$ is $\mathrm{PH}$ on any non-empty intersection $U_{i} \cap U_{j} \neq \emptyset$. The support of $S$ and the polar locus of $S$ are defined by:

$$
\operatorname{supp} S:=\left\{z \in N \text { : if } z \in U_{j} \text {, then } v_{j} \text { is not PH at } z\right\},
$$

and

$$
\operatorname{pol} S:=\left\{z \in N \text { : if } z \in U_{j} \text { then } v_{j}(z)=-\infty\right\} .
$$

The compatibility condition assures that that above sets are well defined. Moreover, since $\mathrm{PH}$ functions are never $-\infty$, we have pol $S \subset \operatorname{supp} S$.

Let $M$ be another complex manifold, possibly of dimension different from that of $N$. If $f: M \rightarrow N$ is a holomorphic map with $f(M) \not \subset$ pol $S$, then the pull-back $f^{*} S$ is a closed positive $(1,1)$ current defined on $M$ by pulling back the system of local potentials for $S$ to form a system of local potentials on $M$ that define $f^{*} S$; see [19, Appendix A.7] and [9, pp. 330-331] for further details.

Given any closed positive $(1,1)$ current $S$ on $N$ and any piecewise smooth two chain $\sigma$ in $N$ with $\partial \sigma$ disjoint from supp $S$, we can define

$$
\langle\sigma, S\rangle=\int_{\sigma} \eta_{S}
$$

where $\eta_{S}$ is a smooth approximation of $S$ within it's cohomology class in $N-\partial \sigma$; see 4. pages 382-385]. The resulting number $\langle\sigma, S\rangle$ will depend only on the cohomology class of $S$ and the homology class of $\sigma$ within $H_{2}(N, \partial \sigma)$. If $\sigma$ is a holomorphic chain, then this pairing simplifies to be the integral of the measure 10 over $\sigma$.

The following invariance property is useful:

Proposition 3.1. Suppose that $S$ is a closed positive $(1,1)$ current on $N$ and $f: M \rightarrow N$ is holomorphic, with $f(M) \not \subset$ pol $S$. If $\sigma$ is a piecewise smooth two chain in $M$ with $\partial \sigma$ disjoint from $\operatorname{supp} f^{*} S$, then $\left\langle f_{*} \sigma, S\right\rangle=\left\langle\sigma, f^{*} S\right\rangle$.

Notice that $H_{2}\left(\mathbb{C P}^{2}\right)$ is generated by the class of any complex projective line $L \subset \mathbb{C P}^{2}$. Since $S$ is non-trivial, $\langle L, S\rangle \neq 0$, so that after an appropriate rescaling, we can assume that $\langle L, S\rangle=1$. This normalization is satisfied by the Green's Current $T$ that was defined in $\$ 2$,

Definition 3.2. Let $S$ be a normalized closed positive $(1,1)$ current on $\mathbb{C P}^{2}$ and let $\gamma$ be a piecewise smooth closed curve in $\mathbb{C P}^{2} \backslash \operatorname{supp}(S)$. We define the linking number $l k(\gamma, S)$ by

$$
l k(\gamma, S):=\langle\Gamma, S\rangle(\bmod 1)
$$

where $\Gamma$ is any piecewise smooth two chain with $\partial \Gamma=\gamma$.

Unlike linking numbers between closed loops in $\mathbb{S}^{3}$, it is often the case that $\langle\Gamma, S\rangle \notin \mathbb{Z}$, resulting in non-zero linking numbers $(\bmod 1)$.

\footnotetext{
${ }^{1}$ This measure is well defined, since $\partial \sigma$ is disjoint from $\operatorname{supp} S \supset \operatorname{pol} S$.
} 
Proposition 3.3. If $\gamma_{1}$ and $\gamma_{2}$ are homologous in $H_{1}\left(\mathbb{C P}^{2} \backslash \operatorname{supp} S\right)$, then $l k\left(\gamma_{1}, S\right)$ $=l k\left(\gamma_{2}, S\right)$.

Moreover, since the pairing $\langle\cdot, S\rangle$ is linear in the space of chains $\sigma$ (having $\partial \sigma$ disjoint from supp $S$ ), the linking number descends to a homomorphism:

$$
l k(\cdot, S): H_{1}\left(\mathbb{C P}^{2} \backslash \operatorname{supp} S\right) \rightarrow \mathbb{R} / \mathbb{Z}
$$

Similarly, $l k(\cdot, S): H_{1}(\Omega) \rightarrow \mathbb{R} / \mathbb{Z}$ for any open $\Omega \subset \mathbb{C P}^{2} \backslash \operatorname{supp} S$.

Theorem 3.4. Suppose that $f: \mathbb{C P}^{2} \rightarrow \mathbb{C P}^{2}$ is a holomorphic endomorphism and $\Omega \subset U(f)$ is contained in a union of basins of attraction of attracting periodic points for $f$. If there are $c \in H_{1}(\Omega)$ with linking number $l k(c, T) \neq 0$ arbitrarily close to 0 in $\mathbb{Q} / \mathbb{Z}$, then $H_{1}(\Omega)$ is infinitely generated.

\section{Proof of the main Result}

We recall the characterization of Axiom-A polynomial skew products from [12, $\S 8]$. (For the actual definition of Axiom-A, see, for example, [12, Def. 8.1].) Let $A_{p}$ be the set of attracting periodic points of $p$ and consider the following postcritical sets:

$$
\begin{aligned}
D_{p} & :=\overline{\bigcup_{n \geq 1} p^{n} C_{p}}, \text { where } C_{p}=\left\{z: p^{\prime}(z)=0\right\}, \\
D_{J_{p}} & :=\overline{\bigcup_{n \geq 1} f^{n} C_{J_{p}}}, \text { where } C_{J_{p}}:=\left\{(z, w): z \in J_{p}, q_{z}^{\prime}(w)=0\right\}, \\
D_{A_{p}} & :=\overline{\bigcup_{n \geq 1} f^{n} C_{A_{p}}}, \text { where } C_{A_{p}}:=\left\{(z, w): z \in A_{p}, q_{z}^{\prime}(w)=0\right\},
\end{aligned}
$$

and

$$
D_{\Pi}:=\overline{\bigcup_{n \geq 1} f_{\Pi}^{n} C_{\Pi}}, \text { where } C_{\Pi}:=\left\{\lambda: f_{\Pi}^{\prime}(\lambda)=0\right\}
$$

Let

$$
J_{A_{p}}=\overline{\bigcup_{z \in A_{p}}\{z\} \times J_{z}} .
$$

The following appears as [12, Cor. 8.3]:

Proposition 4.1. A polynomial skew product $f=(p, q)$ is Axiom- $A$ on $\mathbb{C P}^{2}$ if and only if:

(1) $D_{p} \cap J_{p}=\emptyset$;

(2) $D_{J_{p}} \cap J_{2}=\emptyset$;

(3) $D_{A_{p}} \cap J_{A_{p}}=\emptyset$, and

(4) $D_{\Pi} \cap J_{\Pi}=\emptyset$.

In particular, if $f$ is Axiom-A, then

- every Fatou component of $p$ (respectively $f_{\Pi}$ ) is the basin of attraction of an attracting cycle whose immediate basin contains a critical point from $C_{p}$ (respectively $C_{\Pi}$ ), and

- $C_{z} \cap J_{z}=\emptyset$ over every $z \in A_{p} \cup J_{p}$. 
We can describe the fiberwise dynamics as follows: Let $L:=\{W=0\}$ be the horizontal projective line. The vertical projection $\pi(z, w)=(z, 0)$ induces a rational map $\pi: \mathbb{C P}^{2} \rightarrow L$ whose only point of indeterminacy is $[0: 1: 0]$ (which blows up under $\pi$ to the entire line $L$ ). Although $L$ is not invariant under $f$, we can consider the action of $p$ on the line $L$ and we let $\mu_{p}$ be the harmonic measure on $J_{p}$, i.e. $\mu_{p}:=d d^{c} G_{p}(z)$. Within $\mathbb{C P}^{2}$ we have the vertical current $T_{p}:=\pi^{*} \mu_{p}$.

Throughout the remainder of the paper we will denote by $C$ the closure in $\mathbb{C P}^{2}$ of the "vertical critical points" $\left\{(z, w): q_{z}^{\prime}(w)=0\right\}$. It is an algebraic curve of degree $d-1$ with $C_{z}=C \cap(z \times \mathbb{C})$ and $C \cap \Pi=C_{\Pi}$. Since $q_{z}(w)=w^{d}+O_{z}(w)$, we have that $[0: 1: 0] \notin C$. Therefore, $\pi: C \rightarrow L$ is a branched covering of degree $\operatorname{deg} C=d-1$.

Proposition 4.2. If $f$ is a polynomial skew product with $J_{z}$ connected for every $z \in J_{p}$, then:

(1) For any piecewise-smooth two-chain $\Gamma \subset C$, we have $\langle\Gamma, T\rangle=\left\langle\Gamma, T_{p}\right\rangle$.

(2) Let $U$ be any Fatou component $U$ of $p$ and $C_{U}:=\pi_{\mid C}^{-1}(U)$. The sequence of restrictions $\left\{f_{\mid C_{U}}^{n}\right\}$ is a normal family.

Proof. It suffices to consider any irreducible component $\mathcal{C}$ of $C$, which we parameterize by its normalization $\rho: \widehat{\mathcal{C}} \rightarrow \mathcal{C}$; see [5]. Since $\mathcal{C}$ has dimension $1, \widehat{\mathcal{C}}$ is a compact Riemann surface. The following diagram summarizes the maps $\rho$ and $\pi$ :

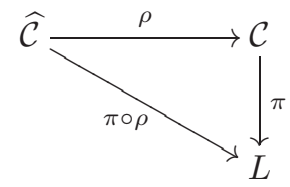

Since $[0: 1: 0] \notin \mathcal{C}$, we need only consider $\mathcal{C}$ within two systems of affine coordinates: the original affine coordinates $(z, w)$ and the coordinates $t=T / Z$ and $u=W / Z$, defined in a neighborhood $\Omega$ of $[1: 0: 0]$. In the $(z, w)$ coordinates, the Green's current is given by $T=\frac{1}{2 \pi} d d^{c} G(z, w)$ with

$$
G(z, w)=G_{z}(w)+G_{p}(z) .
$$

Meanwhile, in the $(t, u)$ coordinates, it is given by $T=\frac{1}{2 \pi} d d^{c} G_{\Omega}(t, u)$ with

$$
G_{\Omega}(t, u)=G_{t}(u)+G_{p}^{\#}(t) .
$$

Here, $G_{p}^{\#}(t)$ is obtained by extending $G_{p}(1 / t)-\log (1 / t)$ continuously through $t=0$ and $G_{t}(u)$ is the PSH extension of $G_{z}(w)$ described in [12, Lemma 6.3]. One has that $(0, u) \in K_{\Pi}$ if and only if $G_{0}(u)=0$ and, for $t \neq 0,(t, u) \in K_{1 / t}$ if and only if $G_{t}(u)=0$.

Because $J_{z}$ is connected for every $z \in J_{p}$, Proposition 6.4 from [12] gives that $C_{z} \subset K_{z}$ for every $z \in \mathbb{C}$ and $C_{\Pi} \subset K_{\Pi}$. Therefore, if $(z, w) \in \mathcal{C} \cap \mathbb{C}^{2}$, we have that $G_{z}(w)=0$ and (4) gives that the restriction of $G$ to $\mathcal{C} \cap \mathbb{C}^{2}$ coincides with $G_{p}(z)$. Similarly, using (5), the restriction of $G_{\Omega}$ to $\mathcal{C} \cap \Omega$ is just $G_{p}^{\#}(t)$.

The above calculations give

$$
\rho^{*} T=\rho^{*} T_{p}=\rho^{*} \pi^{*} \mu_{p} .
$$

\footnotetext{
${ }^{2}$ In other words, $\left\{f^{n} \circ \rho\right\}$ is a normal family for any parameterization $\rho$ of $C_{U}$ (see below).
} 
Let $\Gamma \subset \mathcal{C}$ be any piecewise smooth two chain and $\widehat{\Gamma} \subset \widehat{\mathcal{C}}$ its lift to the normalization. Then,

$$
\langle\Gamma, T\rangle=\left\langle\widehat{\Gamma}, \rho^{*} T\right\rangle=\left\langle\widehat{\Gamma}, \rho^{*} T_{p}\right\rangle=\left\langle\Gamma, T_{p}\right\rangle .
$$

There is a general principle that for any complex manifold $M$ and holomorphic map $\phi: M \rightarrow \mathbb{C P}^{2}$, the family $\left\{f^{n} \circ \phi\right\}$ is normal in a neighborhood of $x \in M$ if and only if $x \in M \backslash \operatorname{supp} \phi^{*} T$; see [3] and also [12, p. 409].

In our situation, it follows that

$$
f^{n} \circ \rho: \widehat{\mathcal{C}} \rightarrow \mathbb{C P}^{2}
$$

is normal at $x \in \widehat{\mathcal{C}}$ if and only if $x \in \widehat{\mathcal{C}} \backslash \operatorname{supp}\left(\rho^{*} T_{p}\right)$. Since $G_{p}$ is harmonic outside of $J_{p}$, we have that $\mathcal{C}_{U}$ is disjoint from supp $T_{p}$ and, hence, that $\hat{\mathcal{C}}_{U}$ is disjoint from $\operatorname{supp}\left(\rho^{*} T_{p}\right)$. Therefore, $f^{n} \circ \rho$ is normal on $\hat{\mathcal{C}}_{U}$.

Corollary 4.3. Suppose that $f$ is Axiom-A and satisfies the hypotheses of Proposition $4.2, z_{0}$ is an attracting periodic point for $p$ (possibly $z_{0}=\infty$ ), and $U$ is any component of the immediate basin for $z_{0}$. Then, $C_{U}$ is in the immediate basins for some attracting periodic points of $f$ within $z=z_{0}$.

Proof. Since $f$ is Axiom-A, $\mathcal{C}_{z_{0}} \subset \mathcal{C}_{U}$ is in the immediate basins of attraction for some attracting periodic points of $f_{\left\{z=z_{0}\right\}}$. Because the line $z=z_{0}$ is transversely attracting, these periodic points are also attracting for $f$ in $\mathbb{C P}^{2}$, giving an open subset of $\mathcal{C}_{U}$ that is in these immediate basins. Since $\left\{f^{n} \circ \rho\right\}$ is normal on $\rho^{-1}\left(\mathcal{C}_{U}\right)$, all of $\mathcal{C}_{U}$ is in the union of these immediate basins.

Proof of Theorem 1.2. Case 1: $f$ is connected:

We begin with Fatou components for $f$ that are bounded in $\mathbb{C}^{2}$, i.e. those on which $G(z, w) \equiv 0$.

Since $J_{p}$ is connected and $p$ is hyperbolic, the Fatou set $U(p)$ consists of the basins of attraction of finitely many attracting periodic orbits together with the basin of attraction for the superattracting fixed point at $z=\infty$. Moreover, each component of these basins is conformally equivalent to the open unit disc $\mathbb{D}$. For simplicity of exposition, we suppose that each attracting periodic orbits for $p$ is a fixed point (otherwise, one can pass to an appropriate iterate of $f$ ).

Let $z_{0} \in \mathbb{C}$ be an attracting fixed point of $p$ and let $U_{0}$ be the component of its basin of attraction that contains $z_{0}$.

Let $x$ and $y$ denote points in $\mathbb{C}^{2}$.

Since $f$ is Axiom-A, $J_{z_{0}}$ is a hyperbolic set, having a local stable manifold $W_{\text {loc }}^{s}\left(J_{z_{0}}\right)$ formed as the union of stable curves $W_{\text {loc }}^{s}(x)$ of points $x \in J_{z_{0}}$ with each $W_{\text {loc }}^{s}(x)$ being holomorphic. These stable curves satisfy the invariance

$$
f\left(W_{\mathrm{loc}}^{s}(x)\right) \subset W_{\mathrm{loc}}^{s}(f(x)) .
$$

They are transverse to the vertical line $\left\{z=z_{0}\right\}$ since it is the unstable direction of $J_{z_{0}}$. Hence, there is a sufficiently small $\epsilon>0$ so that each $W_{\text {loc }}^{s}(x)$ is the graph of a holomorphic function over $z \in \mathbb{D}_{\epsilon}\left(z_{0}\right):=\left\{\left|z-z_{0}\right|<\epsilon\right\}$. In other words, this describes $W_{\text {loc }}^{s}\left(J_{z_{0}}\right)$ as a holomorphic motion (see, for example, [10, §5.2]) over the open disc $z \in \mathbb{D}_{\epsilon}\left(z_{0}\right)$.

Because $\left.f\right|_{z=z_{0}}:\left\{z_{0}\right\} \times \mathbb{C} \rightarrow\left\{z_{0}\right\} \times \mathbb{C}$ is hyperbolic, its Fatou set consists of the basins of attraction of finitely many attracting periodic points (including $w=\infty)$. We will use some hyperbolic theory to show that if $z \in \mathbb{D}_{\epsilon}\left(z_{0}\right)$, then either 
$(z, w) \in W_{\mathrm{loc}}^{s}\left(J_{z_{0}}\right)$ or $(z, w)$ is in the basin of one of these finitely many attracting periodic points for $\left.f\right|_{z=z_{0}}$. Because most classical treatments of hyperbolic theory are for diffeomorphisms, we appeal to the discussion for endomorphisms that appears in [11.

The natural extension of $J_{z_{0}}$ is

$$
\widehat{J}_{z_{0}}=\left\{\left(x_{i}\right)_{i \leq 0}: x_{i} \in J_{z_{0}} \text { and } f\left(x_{i}\right)=x_{i+1}\right\} .
$$

Associated to any prehistory $\hat{x} \in \widehat{J}_{z_{0}}$ is a local unstable manifold $W_{\text {loc }}^{u}(\hat{x})$, which, in this case, is just an open disc in the vertical line $z=z_{0}$.

We will now verify that $\widehat{J}_{z_{0}}$ has a local product structure; see Definition 2.2 from [11]. If $W_{\text {loc }}^{s}(x) \cap W_{\text {loc }}^{u}(\hat{y})$ is non-empty for some $x \in J_{z_{0}}$ and $\hat{y} \in \widehat{J}_{z_{0}}$, then $W_{\text {loc }}^{s}(x) \cap W_{\text {loc }}^{u}(\hat{y})=x$, since $W_{\text {loc }}^{u}(\hat{y})$ is contained in the vertical line $\left\{z=z_{0}\right\}$ and $W_{\text {loc }}^{s}(x)$ is the graph of a function of $z$. In particular, the intersection is a unique point. Moreover, the unique prehistory $\hat{x}$ of $x$ satisfying that $x_{i} \in W_{\text {loc }}^{u}\left(f^{i}(\hat{y})\right)$ for all $i \leq 0$ is in $\widehat{J}_{z_{0}}$, since every local unstable manifold lies in $\left\{z=z_{0}\right\}$.

Because $\widehat{J}_{z_{0}}$ has a local product structure, Corollary 2.6 from 11 gives that there is a neighborhood $V$ of $J_{z_{0}}$ in $\mathbb{C}^{2}$ so that if $f^{n}(z, w) \in V$ for all $n \geq 0$, then $(z, w) \in W_{\text {loc }}^{s}(x)$ for some $x \in J_{z_{0}}$. If $z \in \mathbb{D}_{\epsilon}\left(z_{0}\right)$, then $f^{n}(z, w)$ must converge to the line $\left\{z=z_{0}\right\}$ since $\mathbb{D}_{\epsilon}\left(z_{0}\right) \subset U_{0}$. Existence of the neighborhood $V$ implies that $(z, w)$ is either in $W_{\text {loc }}^{s}\left(J_{z_{0}}\right)$ or is in the basin of attraction for one of the finitely many attracting periodic points for $\left.f\right|_{z=z_{0}}:\left\{z_{0}\right\} \times \mathbb{C} \rightarrow\left\{z_{0}\right\} \times \mathbb{C}$.

We can now see that if $z \in \mathbb{D}_{\epsilon}\left(z_{0}\right)$, then $J_{z}=W^{s}\left(J_{z_{0}}\right) \cap(\{z\} \times \mathbb{C})$. From the preceding paragraph we see that if $(z, w) \notin W_{\text {loc }}^{s}\left(J_{z_{0}}\right)$, then $w \notin J_{z}$. Meanwhile, if $(z, w) \in W_{\text {loc }}^{s}\left(J_{z_{0}}\right)$, then the family of iterates $\left.f^{n}\right|_{\{z\} \times \mathbb{C}}$ cannot be normal at $w$, giving that $w \in J_{z}$.

Repeatedly taking the preimages of $W_{\text {loc }}^{s}\left(J_{z_{0}}\right)$ under $f$ that intersect the vertical line $\left\{z=z_{0}\right\}$, we obtain a stable set $W^{s}\left(J_{z_{0}}\right)$ over all of $U_{0}$. For any $z \in U_{0}$, we have that $(z, w)$ is either in $W^{s}\left(J_{z_{0}}\right)$ or is in the basin attraction for one of the finitely many attracting periodic points of $\left.f\right|_{z=z_{0}}$. We also have $J_{z}=W^{s}\left(J_{z_{0}}\right) \cap(\{z\} \times \mathbb{C})$.

By Corollary 4.3, $C_{U_{0}} \subset U(f)$, so that $C_{U_{0}}$ is disjoint from $W^{s}\left(J_{z_{0}}\right)$. A repeated application of the Inverse Function Theorem, together with (8), allows us to extend (in the parameter $z$ ) the holomorphic motion of $J_{z_{0}}$ from $z \in \mathbb{D}_{\epsilon}\left(z_{0}\right)$ to a holomorphic motion of $J_{z_{0}}$ over all of $U_{0}$. (For details, see the proof of Theorem 6.3 from [8].)

The image of this holomorphic motion is precisely $W^{s}\left(J_{z_{0}}\right)$. By Slodkowski's Theorem [20, it will extend (in the fiber $w$ ) as a holomorphic motion of the entire Riemann Sphere $\mathbb{C P}^{1}$ that is parameterized by $U_{0}$. Therefore, every bounded Fatou component of $f$ lying above $U_{0}$ is given as the holomorphic motion of a Fatou component of $\left.f\right|_{z=z_{0}}$. Each of these is a disc (since $\left.f\right|_{z=z_{0}}$ is hyperbolic with connected Julia set), so every bounded Fatou component lying above $U_{0}$ is homeomorphic to an open ball.

Let $z_{n}$ be an $n$-th preimage under $p$ of $z_{0}$ and let $U_{n}$ be the component of $W^{s}\left(z_{0}\right)$ containing $z_{n}$. We will use induction on $n$ to show that there is a holomorphic motion of $J_{z_{n}}$ parameterized by $z \in U_{n}$ so that:

(1) for every $z \in U_{n}, J_{z}$ is obtained as the motion of $J_{z_{n}}$, and

(2) the motion of any $w \in J_{z_{n}}$ is a precisely stable curve of $w$.

Then, as above, Slodkowski's Theorem will give that every bounded Fatou component of $f$ lying over $U_{n}$ is obtained as the holomorphic motion of some component 
of $K_{z_{n}} \backslash J_{z_{n}}$, parameterized by $z \in U_{n}$. In particular, every such Fatou component will be homeomorphic to an open ball.

The desired holomorphic motion already exists for $n=0$, so we suppose that it exists for $n=k$ in order to prove it for $n=k+1$.

It is sufficient to show that the part $C_{U_{k+1}}$ of the horizontal critical locus that lies above $U_{k+1}$ is in the Fatou set $U(f)$. In that case, the Implicit Function Theorem can be used to lift the entire holomorphic motion of $J_{z_{k}}$ under $f$ to a holomorphic motion of $J_{z_{k+1}}$ parameterized by $U_{k+1}$. The result will automatically satisfy (1) and $(2)$.

Let $\mathcal{C}_{U_{k+1}}$ be some irreducible component of $C_{U_{k+1}}$. By Proposition 4.2 the family of restrictions of iterates $\left\{f_{\mid \mathcal{C}_{U_{k+1}}}^{n}\right\}$ is normal. Therefore, $f^{k+1} \mathcal{C}_{U_{k+1}}$ is either in the Fatou set $U(f)$ or is within the stable curve of a single $x \in J_{z_{0}}$.

Suppose the latter happens. Then, every point of $f^{n+1} \mathcal{C}_{U_{k+1}}$ lying over any $z \in U_{0}$ is in $J_{z}$. Let $\mathcal{C}_{\partial U_{k+1}}$ be the portion of the horizontal critical locus lying over $\partial U_{k+1} \subset J_{p}$ that is in the closure of $\mathcal{C}_{U_{k+1}}$. Lower semi-continuity of the mapping $z \mapsto J_{z}$ (see [12, Prop. 2.1]), combined with continuity of $f^{k+1}$, gives that

$$
f^{k+1} \mathcal{C}_{\partial U_{k+1}} \subset \bigcup_{z \in \partial U_{0}}\{z\} \times J_{z} \subset \overline{\bigcup_{z \in J_{p}}\{z\} \times J_{z}}=J_{2} .
$$

However, since $f$ is Axiom-A, Proposition 4.1 implies that $f^{k+1} \mathcal{C}_{\partial U_{k+1}} \subset D_{J_{p}}$ is disjoint from $J_{2}$. Therefore, we conclude that every component of $C_{U_{k+1}}$ is in the Fatou set.

We now consider the Fatou components for $f$ on which $G(z, w)>0$. Recall that $(z, w) \in W^{s}([0: 1: 0])$ if and only if $G_{z}(w)>0$. Since $J_{z}$ is connected for every $z \in J_{p}$, it follows from Theorem 2.2 that $W^{s}([0: 1: 0])$ is homeomorphic to an open ball.

It remains to consider Fatou components on which $G_{p}(z)>0$ and $G_{z}(w)=0$. Let $U_{\infty} \subset \mathbb{C P}^{1}$ be the basin of attraction of $\infty$ under $p$. Since $J_{p}$ is connected, $U_{\infty}$ is simply connected. For simplicity of exposition, we suppose that $0 \notin U_{\infty}$ (otherwise, one can conjugate $f$ by an appropriate translation in the $z$-coordinate).

Let us work in the system of local coordinates $t=T / Z$ and $u=W / Z$ in which

$$
f(t, u)=\left(\frac{t^{d}}{P(1, t)}, \frac{Q(1, u, t)}{P(1, t)}\right) \equiv\left(r(t), s_{t}(u)\right),
$$

which is a rational skew product. The first coordinate has 0 as a totally-invariant superattracting fixed point, whose basin of attraction $U_{\infty}$ is entirely contained in the copy of $\mathbb{C}$ parameterized by $t$. The Fatou components of $f$ that remain to be studied each have projection under $(t, u) \mapsto t$ lying entirely in $U_{\infty}$.

The line $\Pi$ is given by $t=0$. Since $f$ is Axiom-A, $J_{\Pi}$ is hyperbolic, having a local stable manifold $W_{\mathrm{loc}}^{s}\left(J_{\Pi}\right)$ that is formed as the union of stable curves of points $x \in J_{\Pi}$. Each of these stable curves is transverse to $\Pi$, since $\Pi$ is the unstable direction of $J_{\Pi}$. Thus, we can choose $\epsilon>0$ sufficiently small so that $W_{\text {loc }}^{s}\left(J_{\Pi}\right)$ is described as a holomorphic motion of $J_{\Pi}$ that is parameterized by $t \in \mathbb{D}_{\epsilon}(0)$.

Using the same proof as for $\widehat{J}_{z_{0}}$, one can check that $\widehat{J}_{\Pi}$ has a local product structure, so that if $(t, u)$ satisfies $|t|<\epsilon$, then $(t, u)$ is in the basin of attraction for one of the finitely many attracting periodic points of $f_{\Pi}$ (including $u=\infty$, which corresponds to $[0: 1: 0])$. Similarly, if $|t|<\epsilon$, then $J(f) \cap(\mathbb{C} \times\{t\})=$ $W^{s}\left(J_{\Pi}\right) \cap(\mathbb{C} \times\{t\})$. 
By Corollary 4.3 the horizontal critical locus

$$
C_{U_{\infty}}=\left\{(t, u): \frac{\partial s_{t}(u)}{\partial u}=0 \text { and } t \in U_{\infty}\right\}
$$

lies in the Fatou set for $f$. As before, the Implicit Function Theorem can be used repeatedly to extend the holomorphic motion of $J_{\Pi}$ to one that is parameterized by all of $U_{\infty}$. Then, Slodkowski's Theorem can be used to extend this holomorphic motion in the fiber $w$. Each of the Fatou components of $f$ that lies over $U_{\infty}$ (other than $\left.W^{s}([0: 1: 0])\right)$ is then a holomorphic motion of a bounded Fatou component of the polynomial map $f_{\Pi}$, parameterized by the simply connected domain $U_{\infty}$. This gives that each such component is homeomorphic to an open bidisc.

Case 2: $f$ is disconnected:

If $J_{z}$ is disconnected for any $z \in J_{p}$, then Theorem 2.2 gives that $H_{1}\left(W^{s}([0: 1: 0])\right)$ is infinitely generated. It remains to consider the case that $J_{z}$ is connected for every $z \in J_{p}$ and $J_{p}$ is disconnected.

Let $U_{\infty}$ be the basin of attraction of $z=\infty$ for $p(z)$. Corollary 4.3 gives that each of the irreducible components of $C_{U_{\infty}}=\pi_{\mid C}^{-1}\left(U_{\infty}\right)$ is in the immediate basin of attraction $W_{0}^{s}\left(\zeta_{i}\right)$ of one of the finitely many attracting periodic points

$$
\zeta_{1}:=\left\{\zeta_{1}^{1}, \ldots, \zeta_{1}^{n_{1}}\right\}, \ldots, \zeta_{m}:=\left\{\zeta_{m}^{1}, \ldots, \zeta_{m}^{n_{m}}\right\}
$$

of $f_{\Pi}$.

The proof of Proposition 4.2 from 8 s shows how to generate a sequence of piecewise smooth one-cycles $v_{n} \subset U_{\infty}$ bounding regions $\Upsilon_{n} \subset \mathbb{C}$ with the property that $\left\langle\Upsilon_{n}, \mu_{p}\right\rangle \rightarrow 0$. Perturbing the $v_{i}$ slightly (if needed), we can suppose that none of them lie on the finitely many critical values of $\pi: C \rightarrow L$. For each $i$, we let

$$
\gamma_{i}:=\pi^{-1}\left(v_{i}\right) \text { and } \Gamma_{i}:=\pi^{-1}\left(\Upsilon_{i}\right),
$$

so that each $\gamma_{i}$ is a finite union of closed loops in $C_{U_{\infty}}$ bounded by a piecewise smooth chain $\Gamma_{i}$. Moreover, $\pi: \Gamma_{i} \rightarrow \Upsilon_{i}$ is a ramified cover of degree $d-1$.

Proposition 4.2 gives $\left\langle\Gamma_{i}, T\right\rangle=\left\langle\Gamma_{i}, T_{p}\right\rangle$ and the invariance properties of $\langle\cdot, \cdot\rangle$ from Proposition 3.1 give

$$
\left\langle\Gamma_{i}, T_{p}\right\rangle=\left\langle\Gamma_{i}, \pi^{*} \mu_{p}\right\rangle=\left\langle\pi_{*} \Gamma_{i}, \mu_{p}\right\rangle=(d-1)\left\langle\Upsilon_{i}, \mu_{p}\right\rangle
$$

Since $\left\langle\Upsilon_{i}, \mu_{p}\right\rangle \rightarrow 0$, we have that

$$
l k\left(\gamma_{i}, T\right)=\left\langle\Gamma_{i}, T\right\rangle(\bmod 1)=(d-1)\left\langle\Upsilon_{i}, \mu_{p}\right\rangle(\bmod 1) \rightarrow 0(\bmod 1) .
$$

Theorem 3.4 gives that the first homology of $\bigcup_{i} W_{0}^{s}\left(\zeta_{i}\right)$ is infinitely generated. Hence, at least one of the finitely many Fatou components from this union has infinitely generated homology.

Remark 4.4. We will now discuss the question of whether Theorem 1.2 holds without the hypothesis that $f$ be Axiom-A. To see that some condition on hyperbolicity is needed, consider the disconnected product mapping

$$
f(z, w)=\left(z^{2}-6, w^{2}+i\right)
$$

which has that $J_{p}$ is a Cantor set and that $J_{z}$ is a (connected) dendrite for every $z \in \mathbb{C}$. Since $J_{z}$ is connected for every $z \in J_{p}$, Theorem 2.2 gives that $W^{s}([0: 1: 0])$ is homeomorphic to an open ball. Moreover, the closure of $W^{s}([0: 1: 0])$ is all of $\mathbb{C P}^{2}$, giving that it is the only Fatou component for $f$. 
Meanwhile, the author does not presently know of any example of a connected polynomial skew product having any Fatou component that is not homeomorphic to an open ball.

One can somewhat weaken the hypothesis that $f$ be Axiom-A and still have the proof of Theorem 1.2 that is presented in this section hold. (We leave the AxiomA condition in the statement of Theorem 1.2. in order to keep it simple.) More specifically:

If $J_{z}$ is disconnected for some $z \in J_{p}$, then no hypothesis is needed in order to determine that $W^{s}([0: 1: 0])$ has infinitely generated first homology; see Example 5.5 .

If $J_{z}$ is connected for every $z \in J_{p}$, but $J_{p}$ is disconnected, we only need that $f_{\Pi}$ has at least one attracting periodic orbit $\zeta_{0}$. Then, some irreducible component of $C_{U_{\infty}}$ will be in $W_{0}^{s}\left(\zeta_{i}\right)$ and the technique from Case 2 can be applied to that component.

If $f$ is connected, then one need only assume that $J_{p}, J_{\Pi}$, and $J_{A_{p}}$ be hyperbolic. In this case, a slight modification of the proof of Theorem 1.2 is needed to address the issue of bounded Fatou components lying over a preimage $U_{k+1}$ of some immediate basin $U_{0}$ for $p$. Without assuming that $f$ is Axiom-A, $f^{k+1} \mathcal{C}_{U_{k+1}}$ could potentially land in the stable curve $W^{s}(x)$ of some $x \in J_{z_{0}}$. However, one can still use the Implicit Function Theorem to pull back all but finitely many leaves from the holomorphic motion $W^{s}\left(J_{z_{k}}\right)$. By the Lambda Lemma (see [13] and [14), this holomorphic motion (that is missing a few leaves) can be extended to a holomorphic motion of all of $J_{z_{k+1}}$. One then proceeds using Slodkowski's Theorem, as in the original proof.

\section{EXAMPLES}

We conclude this note by interpreting some of the examples of Axiom-A polynomial skew products from [12, [2], and 21] in the context of Theorem 1.2.

Example 5.1. Trivial examples of Axiom-A polynomial skew products exhibiting both behaviors from Theorem 1.2 come from product maps $f(z, w)=(p(z), q(w))$ with $p$ and $q$ hyperbolic and small perturbations thereof.

Example 5.2. The family

$$
f_{a}(z, w)=\left(z^{2}, w^{2}+a z\right)
$$

is considered by DeMarco-Hruska in Section 5.3 from [2. The map $f_{a}$ is Axiom-A if and only if $g_{a}(w):=w^{2}+a$ is hyperbolic [2, Theorem 5.1]. (In that case, $f_{a}$ is in the same hyperbolic component as a product if and only if $g_{a}$ has an attracting fixed point.) Moreover, $J_{p}$ is the unit circle $\{|z|=1\}$ and $J_{e^{i t}}$ is a rotation of angle $t / 2$ of $J_{1}=J\left(g_{a}\right)$; see [2, Lemma 5.5]. Thus, $f_{a}$ is connected if and only if $a$ is in the Mandelbrot set $\mathcal{M}$.

If $a$ is a hyperbolic point from $\mathcal{M}$, then Theorem 1.2 gives that the Fatou set of $f_{a}$ is a union of open balls. Meanwhile, if $a \in \mathbb{C} \backslash \mathcal{M}$, then the basin of attraction $W^{s}([0: 1: 0])$ has infinitely generated first homology. (See also [8, Theorem 6.3], where the assumption that $f_{a}$ be Axiom-A is dropped.)

Example 5.3. Jonsson shows in Example 9.6 from [12] that

$$
f(z, w)=\left(z^{2}-6, w^{2}+3-z\right)
$$


R. K. W. ROEDER

is Axiom- $\mathrm{A}$ on $\mathbb{C P}^{2}$ and is not in the same hyperbolic component as any product mapping. There are two reasons why $f$ is disconnected:

(1) $p(z)=z^{2}-6$ has a disconnected Julia set, and

(2) $J_{z_{0}}$ is disconnected over the repelling fixed point $z_{0}=-2 \in J_{p}$.

Since $J_{z_{0}}$ is disconnected, Theorem 2.2 gives that $W^{s}\left(\left[\begin{array}{llll}0 & : & : & 0\end{array}\right]\right)$ has infinitely generated first homology.

The action on the line at infinity is given by $u \mapsto u^{2}$, where $u=w / z$, so that $f$ has two superattracting fixed points on that line: $[0: 1: 0]$ and $[1: 0: 0]$. Even though $J_{p}$ is disconnected, the proof of Case 2 from Theorem 1.2 does not give that $W^{s}([1: 0: 0])$ has infinitely generated first homology, because some points on the horizontal critical locus $C=\{w=0\}$ have orbits escaping to $[0: 1: 0]$. (We do not know if $W^{s}([1: 0: 0])$ has complicated first homology.)

Example 5.4. The following example by Sumi appears in [21, Remark 4.13]. Let

$$
f(z, w)=\left(p(z), w^{2^{n}}+\left(\frac{z+\sqrt{R}}{2 \sqrt{R}}\right) t_{n, \epsilon}(w)\right),
$$

where $R, \epsilon>0, n \in \mathbb{N}, p_{R}(z)=z^{2}-R, p=p_{R}^{n}, h_{\epsilon}(w)=(w-\epsilon)^{2}-1+\epsilon$, and $t_{n, \epsilon}(w)=h_{\epsilon}^{n}(w)-w^{2^{n}}$. For appropriate choices of $\epsilon$ sufficiently small, $R$ sufficiently large, and $n$ sufficiently large and even, one has that $f$ is Axiom-A, not in the same hyperbolic component as any product mapping, with $J_{p}$ a Cantor set and $J_{z}$ connected over every $z \in \mathbb{C}$.

The action of $f$ on the line at infinity is given by $u \mapsto u^{2^{n}}$, thus $f$ has two superattracting fixed points $[0: 1: 0]$ and $[1: 0: 0]$ on the line at infinity. Since $J_{z}$ is connected for every $z \in \mathbb{C}$ and $J_{p}$ is disconnected, the proof of Theorem 1.2 gives that $W^{s}([1: 0: 0])$ has infinitely generated first homology. Meanwhile, Theorem 2.2 gives that $W^{s}([0: 1: 0])$ is homeomorphic to an open ball.

Example 5.5. Jonsson shows in Example 9.7 from [12] that

$$
f(z, w)=\left(z^{2}-2, w^{2}+2(2-z)\right)
$$

has $J_{2}$ connected, but is not a connected skew product according to Definition 1.1 since $J_{z}$ is disconnected for $z=-2$. This skew product is not Axiom-A because $p(z)$ has its critical point in the Julia set $J_{p}=[-2,2]$. However, Theorem 2.2 can still be applied to see that $W^{s}([0: 1: 0])$ has infinitely generated first homology.

\section{ACKNowledgments}

Many of the techniques and ideas from this paper were originally inspired by John H. Hubbard during discussions that we had when I was his student (5 years ago). I have also benefited greatly from discussions with Laura DeMarco, Mattias Jonsson, Suzanne Hruska, Lex Oversteegen, and Rodrigo Perez.

The anonymous referee has provided many helpful comments leading to substantial improvements in the paper.

\section{REFERENCES}

[1] Eric Bedford and Mattias Jonsson. Dynamics of regular polynomial endomorphisms of $\mathbf{C}^{k}$. Amer. J. Math., 122(1):153-212, 2000. MR.1737260 (2001c:32012)

[2] Laura DeMarco and Suzanne Lynch Hruska. Axiom A polynomial skew products of $\mathbb{C}^{2}$ and their postcritical sets. Ergodic Theory Dynam. Systems, 28(6):1749-1779, 2008. MR2465599 (2009m:37132) 
[3] John Erik Fornæss and Nessim Sibony. Complex dynamics in higher dimension. II. In Modern methods in complex analysis (Princeton, NJ, 1992), volume 137 of Ann. of Math. Stud., pages 135-182. Princeton Univ. Press, Princeton, NJ, 1995. MR1369137 (97g:32033)

[4] Phillip Griffiths and Joseph Harris. Principles of algebraic geometry. Wiley-Interscience [John Wiley \& Sons], New York, 1978. Pure and Applied Mathematics. MR507725 (80b:14001)

[5] Robert C. Gunning. Introduction to holomorphic functions of several variables. Vol. I. The Wadsworth \& Brooks/Cole Mathematics Series. Wadsworth \& Brooks/Cole Advanced Books \& Software, Pacific Grove, CA, 1990. Function theory. MR 1052649 (92b:32001a)

[6] Stefan-M. Heinemann. Julia sets for holomorphic endomorphisms of $\mathbf{C}^{n}$. Ergodic Theory Dynam. Systems, 16(6):1275-1296, 1996. MR1424399 (97j:32020)

[7] Stefan-M. Heinemann. Julia sets of skew products in $\mathbf{C}^{2}$. Kyushu J. Math., 52(2):299-329, 1998. MR 1645451 (99g:32048)

[8] Suzanne Lynch Hruska and Roland K.W. Roeder. Topology of Fatou components for endomorphisms of $\mathbb{C P}^{k}$ : linking with the green's current. Fundamenta Mathematica, 210(1):73-98, 2010. MR 2720217

[9] John H. Hubbard and Peter Papadopol. Superattractive fixed points in $\mathbf{C}^{n}$. Indiana Univ. Math. J., 43(1):321-365, 1994. MR1275463 (95e:32025)

[10] John Hamal Hubbard. Teichmüller theory and applications to geometry, topology, and dynamics. Vol. 1. Matrix Editions, Ithaca, NY, 2006. Teichmüller theory with contributions by Adrien Douady, William Dunbar, Roland Roeder, Sylvain Bonnot, David Brown, Allen Hatcher, Chris Hruska and Sudeb Mitra, with forewords by William Thurston and Clifford Earle. MR 2245223 (2008k:30055)

[11] Mattias Jonsson. Dynamical studies in several complex variables. PhD thesis, Royal Institute of Technology, 1997. MR2715249

[12] Mattias Jonsson. Dynamics of polynomial skew products on C $^{2}$. Math. Ann., 314(3):403-447, 1999. MR1704543(2000f:32025)

[13] M. Yu. Lyubich. Some typical properties of the dynamics of rational mappings. Uspekhi Mat. Nauk, 38(5(233)):197-198, 1983. MR 718838 (85f:58063)

[14] R. Mañé, P. Sad, and D. Sullivan. On the dynamics of rational maps. Ann. Sci. École Norm. Sup. (4), 16(2):193-217, 1983. MR732343 (85j:58089)

[15] John Milnor. Dynamics in one complex variable, volume 160 of Annals of Mathematics Studies. Princeton University Press, Princeton, NJ, third edition, 2006. MR 2193309 (2006g:37070)

[16] Roland K. W. Roeder. A degenerate Newton's map in two complex variables: linking with currents. J. Geom. Anal., 17(1):107-146, 2007. MR.2302877(2009j:37068)

[17] Olivier Sester. Hyperbolicité des polynômes fibrés. Bull. Soc. Math. France, 127(3):393-428, 1999. MR1724402 (2000j:37057)

[18] Olivier Sester. Combinatorial configurations of fibered polynomials. Ergodic Theory Dynam. Systems, 21(3):915-955, 2001. MR1836438 (2002d:37074)

[19] Nessim Sibony. Dynamique des applications rationnelles de $\mathbf{P}^{k}$. In Dynamique et géométrie complexes (Lyon, 1997), volume 8 of Panor. Synthèses, pages ix-x, xi-xii, 97-185. Soc. Math. France, Paris, 1999. MR1760844 (2001e:32026)

[20] Zbigniew Slodkowski. Extensions of holomorphic motions. Ann. Scuola Norm. Sup. Pisa Cl. Sci. (4), 22(2):185-210, 1995. MR:1354904 (96k:30026)

[21] Hiroki Sumi. Dynamics of postcritically bounded polynomial semigroups III: classification of semi-hyperbolic semigroups and random Julia sets which are Jordan curves but not quasicircles. Ergodic Theory Dynam. Systems, 30(6):1869-1902, 2010.

[22] Tetsuo Ueda. Fatou sets in complex dynamics on projective spaces. J. Math. Soc. Japan, 46(3):545-555, 1994. MR1276837 (95d:32030)

iUPUi Department of Mathematical Sciences, LD Building, Room 270, 402 North BlackFord Street, Indianapolis, Indiana 46202-3267

E-mail address: rroeder@math.iupui.edu 